Arch. Vet. Scienc. 3(1):51-55, 1998

Printed in Brazil

\title{
ESTUDO DO TIPO DE HEMOGLOBINA COMO AUXILIAR NA SELEÇÃO DE OVINOS RESISTENTES E SUSCEPTÍVEIS AOS HELMINTOS GASTRINTESTINAIS*
}

\author{
CRISTINA SANTOS SOTOMAIOR ${ }^{1}$; VANETE THOMAZ-SOCCOL ${ }^{2}$ \\ ${ }^{1}$ Curso de Pós-Graduação em Ciências Veterinárias - Universidade Federal do Paraná. ${ }^{2}$ Laboratório de \\ Parasitologia Veterinária Departamento de Patologia Básica, UFPR. Centro Politécnico - Jardim das Américas \\ CEP 81531-990 - Curitiba - PR.
}

\begin{abstract}
Sheep production has been showing, these days, a greater economical and social importance in the State of Paraná, Brazil. The gastrointestinal nematodes are the major problem causing widespread clinical disease and productivity losses, mainly when associated with the high intensive husbandry system and the critic situation of anthelminthic resistance. In order to find alternative or complementary control methods to combat parasitism, one sheep flock of 64 ewes and 77 lambs was monitored to study whether there is a relationship between haemoglobin type and genetic resistance to gastrointestinal nematodes. Haemoglobin typing was performed by starch gel electrophoresis. In 141 animals examined, it was found that $80,14 \%$ were haemoglobin BB type; $17,02 \%$ were $\mathrm{AB}$ type; and only $2,84 \%$ showed a genotype AA, resulting in an allelic frequency of 0,11 and 0,89 for the alleles $\mathrm{A}$ and $\mathrm{B}$, respectively. When these animals were classified into resistant and susceptible to gastrointestinal nematodes, using faecal egg counts, it was observed that this classification was done independently of the haemoglobin type.
\end{abstract}

Key Words: Sheep, haemoglobin type, nematodes, genetic resistance, host selection.

RESUMO - A criação de ovinos vem adquirindo, ao longo do tempo, importância econômica e social no Estado do Paraná. As parasitoses gastrintestinais tornam-se muitas vezes fatores limitantes desta cultura, principalmente quando associadas ao sistema intensivo de criação e à resistência dos parasitas aos diferentes princípios ativos. Com o objetivo de buscar alternativas ao controle da verminose ovina, estudou-se, em um rebanho do município de Curitiba-PR, a relação do tipo de hemoglobina $(\mathrm{Hb})$ com a resistência dos animais aos helmintos gastrintestinais. A determinação do tipo de $\mathrm{Hb}$ foi feita por meio de eletroforese em gel espesso de amido. Dos 141 animais testados, $80,14 \%$ eram do tipo $\mathrm{BB} ; 17,02 \%$ do tipo $\mathrm{AB}$ e apenas $2,84 \%$ dos ovinos eram do genótipo AA, resultando na freqüência alélica de 0,11 e 0,89 para os alelos A e B, respectivamente. Quando estes animais foram classificados em resistentes e susceptíveis aos helmintos gastrintestinais, através da contagem de ovos nas fezes, observou-se que esta classificação ocorreu independente do tipo de $\mathrm{Hb}$.

Palavras-Chave: Ovinos, tipo de hemoglobina, parasitoses gastrintestinais, resistência genética.

\section{Introdução}

A ovinocultura paranaense apresentou, nos últimos anos, um importante aumento no número de animais, após importações da ordem de 187.000 matrizes, realizadas pelo governo do Paraná. Desta forma, o rebanho atual de aproximadamente 630.500 ovinos (SEAB, 1997), passou a ter maior importância econômica e social no Estado.

As parasitoses gastrintestinais são um dos maiores problemas enfrentados por técnicos e criadores. Quando associadas à subnutrição, erros

\footnotetext{
*Trabalho com base na Tese de Mestrado do Curso de PósGraduação em Ciências Veterinárias, UFPR, de Cristina Santos Sotomaior.

*Com auxílio da Coordenação do Aperfeiçoamento do Pessoal de Ensino Superior - CAPES - e do Conselho Nacional do Desenvolvimento Científico e Tecnológico - CNPq. *Correspondência para Profa. Vanete Thomaz-Soccol, Laboratório de Parasitologia Veterinária, Setor de Ciências Biológicas, UFPR, Jardim das Américas, CEP 81531-990 Curitiba, PR. E-mail: vasoccol @ garoupa.bio.ufpr.br.
}

de manejo e ineficiência dos anti-helmínticos, podem se converter em fatores limitantes da produção ovina. Ainda que não se tenha dados oficiais sobre a porcentagem de perdas na ovinocultura paranaense, estima-se que estas sejam altas, por algumas características peculiares à criação neste Estado. Normalmente, os ovinos são criados em áreas pequenas e com superlotação, fazendo com que o alto índice de larvas nas pastagens seja uma fonte de constante contaminação. $\mathrm{Na}$ tentativa de controlar esta situação, esquemas de desverminação múltipla (30 em 30 dias) e supressiva (15 em 15 dias) foram amplamente utilizados, contribuindo para o rápido desenvolvimento da resistência dos parasitas aos diferentes princípios ativos (THOMAZ-SOCCOL et al., 1996; SOUZA, 1997).

O problema de resistência dos nematóides aos anti-helmínticos é também uma preocupação mundial (WALLER, 1994; LE JAMBRE, 1996). Percebendo a iminente falência do sistema de controle de verminose, baseado exclusivamente em 
anti-helmínticos, pesquisadores do mundo todo têm buscado outras alternativas (ALBERS et al., 1987; GILL, 1991). Dentre elas, destaca-se a busca de animais geneticamente mais resistentes aos parasitas gastrintestinais.

Um dos primeiros parâmetros estudados e associados à resistência foi o tipo de hemoglobina. Podem ser identificados três tipos de hemoglobina nos ovinos: AA, AB e BB, determinados geneticamente por dois alelos (EVANS et al., 1956; HUISMAN et al, 1958). Nas décadas de 60 e 70, muitos trabalhos analisaram a associação do tipo de hemoglobina à maior resistência aos parasitas. Todavia, esta possível associação continua sendo questionada em estudos mais recentes.

O presente trabalho teve como objetivo estudar a relação entre tipo de hemoglobina e resistência aos helmintos gastrintestinais, analisando a possibilidade de incluir esta característica em programas de seleção de animais resistentes.

\section{Material e Métodos}

Animais. Os ovinos utilizados no experimento pertenciam à Prefeitura Municipal de Curitiba e estavam alocados nos parques Barigüi e Náutico. Ao todo, foram utilizados 64 ovelhas e 77 cordeiros, filhos dessas ovelhas. As ovelhas eram mestiças Suffolk e tinham de 2 a 6 anos. Inicialmente, as ovelhas foram inseminadas com sêmen de vários carneiros, provenientes de diferentes propriedades, todos da raça Suffolk. No restante do período da estação de monta, as ovelhas foram cobertas por um carneiro mestiço Suffolk.

A seleção das ovelhas e dos cordeiros em resistentes e susceptíveis aos helmintos gastrintestinais foi baseada na contagem de ovos nas fezes, segundo critérios determinados em SOTOMAIOR, 1997.

Determinação do tipo de hemoglobina. A determinação do tipo de hemoglobina foi realizada por meio de eletroforese em gel espesso de amido. A realização técnica compreende seis etapas, descritas a seguir.

Preparação da solução tampão. $O$ tampão utilizado foi o Tris-EDTA-Borato, $\mathrm{pH}$ 8,6 (HUHENS, 1968), diluído na proporção de 1:7 para as cubas, e na proporção de 1:20 para a preparação do gel.

Preparação das amostras de sangue. $O$ sangue das ovelhas e dos cordeiros era colhido da veia jugular externa, em tubos de "vacuntainer" contendo EDTA como anticoagulante. No laboratório, as amostras eram centrifugadas a 2.500 rpm, por 5 minutos. O plasma era desprezado, acrescentando-se o mesmo volume em solução salina a $0,85 \%$. Este procedimento era repetido até o sobrenadante ficar completamente límpido. Após a última centrifugação, provocava-se a hemólise pelo acréscimo de água destilada e agitação vigorosa. Em seguida, era acrescentado $1 / 2$ volume de clorofórmio e centrifugava-se novamente, para separação do estroma. As amostras eram diluídas na proporção de 1:4, a fim de que não houvesse a formação de bandas muito espessas.

Preparação do gel. Para a confecção do gel de amido, utilizou-se a técnica de PETZL e PRIMOPARMO (1979), que trabalha com gel de amido comercial $^{1}$. À solução de tampão, diluída em água na proporção de 1:20, acrescentava-se amido na concentração de 10 \%. Após a homogeneização, a amostra era levada ao forno de microondas, onde o gel era cozido, em potência máxima, por cerca de 12 minutos. O gel era despejado sobre uma placa de acrílico $(21 \mathrm{~cm} \times 21 \mathrm{~cm} \times 1 \mathrm{~cm})$, coberto com uma folha de acetato e com um vidro por cima. A placa com o gel permanecia em temperatura ambiente até o resfriamento e levada à geladeira por $1 \mathrm{a} 2$ horas.

Aplicação das amostras de hemoglobina. As amostras, embebidas em papel Wattman $n^{\circ} 3$ de 1 $\mathrm{cm}^{2}$, eram introduzidas no gel, com o auxílio de uma pinça de ponta fina. Os orifícios, no gel, eram previamente feitos com o auxílio de um pente de 12 fendas verticais espaçadas de $5 \mathrm{~mm}$. Um padrão de hemoglobina A e outro de hemoglobina B sempre eram utilizados em cada gel, como controle.

Migração eletroforética. Uma vez colocadas as amostras, a placa contendo o gel era acoplada ao sistema horizontal de eletroforese. A ponte entre as cubas e a placa era feita com o auxílio de um tecido esponjoso $^{2}$. Esta conexão permitia estabelecer um campo elétrico uniforme, em toda a longitude do suporte. Um gerador de corrente contínua era ligado ao sistema, para permitir a migração dos extratos.

A migração ocorre do pólo negativo para o positivo. A fonte era ligada a $120 \mathrm{~V}$, durante 12 horas. A corrida eletroforética era feita dentro de um refrigerador para evitar a desnaturação da proteína. Após o término da migração, o gel era retirado da placa e cortado longitudinalmente ao meio, sobre o qual era feita a coloração. Coloração. O corante era despejado sobre a parte interna do gel. Em seguida, eram adicionados $3 \mathrm{ml}$ de água oxigenada (HIGASHI e LUBS, 1966). A leitura era feita após alguns minutos, quando as bandas azuladas tornavam-se mais evidentes.

\section{Resultados e Discussão}

A determinação do tipo de hemoglobina mostrou

\footnotetext{
${ }^{1}$ Maizena ${ }^{\circledR}$ - Refinações de Milho Brasil LTDA

${ }^{2}$ Perfex ${ }^{\circledR}$ - Johnson \& Johnson Indústria e Comércio Ltda.
} 
Tipo de Hemoglobina na Seleção de Ovinos Resistentes e Susceptíveis a Helmintos Gastrintestinais

que, das 64 ovelhas, apenas uma apresentou o genótipo $\mathrm{AA}$, seis apresentaram o $\mathrm{AB}$ e 57, o genótipo BB. A freqüência do alelo A aumenta nos cordeiros, onde há três animais AA, 18 com genótipo $\mathrm{AB}$ e 56 com o $\mathrm{BB}$. Isto significa uma freqüência alélica de 0,11 e 0,89 para os alelos A e $\mathrm{B}$, respectivamente, neste rebanho estudado (Tab. 1).

Tabela 1 - Freqüência dos tipos de hemoglobina (AA, AB e BB) das ovelhas e cordeiros, em um rebanho da região de Curitiba - PR.

\begin{tabular}{c|c|c|c|c|c|c|c|c|c}
\cline { 2 - 10 } & \multicolumn{2}{c|}{ AA } & \multicolumn{2}{c|}{ AB } & \multicolumn{2}{c|}{ BB } & TOTAL & $\begin{array}{c}\text { Freqüência } \\
\text { alélica A }\end{array}$ & $\begin{array}{c}\text { Freqüência } \\
\text { alélica B }\end{array}$ \\
\cline { 2 - 11 } & $\mathrm{n}$ & $\%$ & $\mathrm{n}$ & $\%$ & $\mathrm{n}$ & $\%$ & $\mathrm{n}$ & & \\
\hline Ovelhas & 01 & 1,56 & 06 & 9,38 & 57 & 89,06 & 64 & 0,06 & 0,94 \\
\hline Cordeiros & 03 & 3,90 & 18 & 23,39 & 56 & 72,73 & 77 & 0,16 & 0,84 \\
\hline Total & 04 & 2,84 & 24 & 17,02 & 113 & 80,14 & 141 & 0,11 & 0,89 \\
\hline
\end{tabular}

$\mathrm{n}=$ número de animais

A freqüência do tipo de hemoglobina em ovinos criados no município de Santa Maria - RS foi estudada por FAN et al., (1981). Os resultados obtidos por esses autores são próximos aos encontrados no presente trabalho: $4,7 \%$ de ovinos tipo AA; $29,7 \%$ de animais com o genótipo AB; e a maior percentagem $(65,6 \%)$, apresentou a hemoglobina $\mathrm{BB}$. A raça Crioula Lanada, criada no sul do país, também foi avaliada para o tipo de hemoglobina. A freqüência do alelo A foi de 0,33, superior à freqüência encontrada neste trabalho (HENKES et al., 1993).

$\mathrm{Na}$ literatura internacional, há grande variação na freqüência dos alelos A e B, sendo possível distinguir raças com predominância de um ou de outro tipo (EVANS, et al., 1957; HUISMAN, et al., 1958). Para a raça Suffolk, há registro nos EUA de rebanhos com freqüência de 0,85 e
1,00 para o alelo B (WANG, et al., 1991).

No presente trabalho, a seleção dos animais em resistentes e susceptíveis foi feita levando em consideração o número de ovos de helmintos por grama de fezes, por ser este um reflexo direto do parasitismo. Várias hipóteses foram formuladas para determinar o nível de corte entre animais considerados resistentes e susceptíveis (ver SOTOMAIOR, 1997). Em qualquer uma das hipóteses propostas, para a seleção das ovelhas e cordeiros, encontram-se animais com hemoglobina de tipo $\mathrm{AA}, \mathrm{AB}$ e $\mathrm{BB}$ nos grupos considerados resistentes e susceptíveis.

$\mathrm{Na}$ Tab. 2, observam-se as porcentagens dos animais de genótipo $\mathrm{AA}$ e $\mathrm{AB}$, que foram classificados como resistentes ou susceptíveis. Os demais animais de cada grupo são todos de genótipo BB.

Tabela 2 - Percentagem de ovelhas e cordeiros AB e AA em relação ao $\mathrm{n}^{\circ}$ de animais considerados resistentes ou susceptíveis em cada hipótese, em um rebanho da região de Curitiba-PR.

\begin{tabular}{c|c|c|c|c|c|c|c|c}
\multicolumn{2}{c}{$\%$ AB resistente } & \multicolumn{2}{c|}{$\%$ AB susceptível } & \multicolumn{2}{c|}{$\%$ AA resistente } & \multicolumn{2}{c}{$\%$ AA susceptível } \\
\hline Hipótese & Ovelhas & Cordeiros & Ovelhas & Cordeiros & Ovelhas & Cordeiros & Ovelhas & Cordeiros \\
\hline 01 & 12,50 & 26,67 & 14,28 & 23,53 & 6,25 & - & - & 11,76 \\
\hline 02 & 7,14 & 26,67 & 16,67 & 25,00 & - & - & - & 12,50 \\
\hline 03 & - & 33,33 & 20,00 & 22,22 & 11,11 & - & - & 11,11 \\
\hline 04 & 12,50 & 30,77 & 11,11 & 20,00 & - & - & - & 10,00 \\
\hline 05 & 5,56 & 20,00 & 15,38 & 23,53 & - & - & - & 5,88 \\
\hline
\end{tabular}

Vários pesquisadores encontram relação entre o genótipo AA e uma maior resistência aos parasitas gastrintestinais (JILEK e BRADLEY, 1969; ALLONBY e URQUHART, 1976; ALTAIF e DARGIE, 1978; PRESTON e ALLONBY, 1979 a, b; CHIMINAZZO et al., 1996). Estes autores sugerem que, dentro de uma mesma raça, ovinos com genótipo AA são mais resistentes à infecção por helmintos que aqueles de genótipo $\mathrm{AB}$ ou $\mathrm{BB}$.
Estes animais apresentariam menor contagem de ovos de helmintos nas fezes, menor número e tamanho de parasitas adultos.

Algumas hipóteses foram levantadas para explicar esta maior resistência dos animais portadores do genótipo AA. A primeira sugere que a habilidade dos animais de genótipo AA em resistir aos parasitas seria em virtude da maior afinidade deste tipo de hemoglobina ao oxigênio 
(HUISMAN, et al., 1958). Outra hipótese sugere que, devido à habilidade da hemoglobina $\mathrm{A}$ produzir um tipo especial de hemoglobina (hemoglobina tipo C), durante a fase de anemia, os animais portadores deste tipo de hemoglobina seriam mais resistentes aos efeitos das parasitoses (BLUNT e EVANS, 1963; BEALE et al., 1966). Outros trabalhos, por sua vez, defendem a teoria de que as diferenças encontradas quanto à resistência estariam mais ligadas à habilidade dos animais em desenvolverem uma boa resposta imunológica e que o tipo de hemoglobina atuaria somente como um marcador para esta habilidade (CUPERLOVIC, et al., 1978; PRESTON e ALLONBY, 1979).

No presente experimento, a baixa percentagem de animais com genótipo AA não permitiu que fossem feitas correlações entre o tipo de hemoglobina e outros parâmetros indicadores de parasitismo. Porém, a resposta dos animais frente às parasitoses parece ser independente do tipo de hemoglobina. Esta observação é corroborada por outros autores, que não encontraram relação entre tipo de hemoglobina e resistência à verminose (YAZWINSKI et al., 1980; WINDON et al., 1980; KASSAI et al., 1990). Assim sendo, este parâmetro seria de pouca utilidade em um programa de seleção de ovinos resistentes aos helmintos gastrintestinais.

\section{REFERÊNCIAS BIBLIOGRÁFICAS}

ALBERS, G.A.A; GRAY, G.D.; PIPER, L.R.; BARKER, J.S.F.; LE JAMBRE, L.F.; BARGER, I.A. The genetics of resistance and resilience to Haemonchus contortus infection in young Merino sheep. Int. J. Paras. 17(7):1355-1363, 1987.

ALLONBY, E.W.; URQUHART, G.M. A possible relationship between haemonchosis and haemoglobin polymorphism in Merino sheep in Kenya. Res. in Vet. Sci. 20:212-214, 1976.

ALTAIF, K.I.; DARGIE, J.D. Genetic resistance to helminths: The influence of breed and haemoglobin type on the response of sheep to re-infection with Haemonchus contortus. Parasitology. 77:177-187, 1978.

BEALE, D.; LEHMANN, H.; DRURY, A.; TUCKER, E.M. Haemoglobins of sheep. Nature. 209(5028):1099-1102, 1966.

BLUNT, M.H.; EVANS, J.V. Changes in the concentation of potassium in the erythrocytes and in haemoglobin type in Merino sheep under a severe anaemic stress. Nature. 200(4912):12151216, 1963.

CHIMINAZZO, C.; RIBEIRO, L.A.O.; WEIMER, T.; CABRAL, P.C. Correlação dos tipos de hemoglobina com a resitência à verminose em ovinos da raça Corriedale. XV Panvet Abstracts. p. 307, 1996.

CUPERLOVIC, K.; ALTAIF, K.I.; DARGIE, J.D. Genetic resistance to helminths: a possible relationship between haemoglobin type and the immune responses of sheep to non-parasitic antigens. Res. Vet. Sci. 25:125-126, 1978.
EVANS, J.V.; KING, J.W.B.; COHEN, B.L.; HARRIS, H.; WARREN, F.L. Genetics of haemoglobin and blood potassium differences in sheep. Nature. 178(4536):849-850, 1956.

EVANS, J.V.; HARRIS, H.; WARREN, F.L. Haemoglobin types in British breeds of sheep. Biochem. J. 65:42, 1957.

FAN, L.C.R.; MOREIRA, E.C.; FISCHER, R. Freqüência dos tipos de hemoglobina em ovinos adultos no município de Santa Maria. Revista do Centro de Ciências Rurais. 11(1):7-11, 1981.

GILL, H.S. Genetic control of acquired resistance to haemonchosis in Merino lambs. Parasite Immunology. 13:617-628, 1991.

HENKES, L.E.; WEIMER, T.A.; FRANCO, M.H.; MORAES, J.C.F. Genetics characterization of the "Crioula Lanada" sheep. Braz. J. Gen. 16(2):449455, 1993.

HIGASHI, G.I.; LUBS JR, H.A. Quantitative variations of haptoglobins in a caucasian family. J. Med. Genet. 3:281-284, 1966.

HUHENS, E.R. Starch gel electrophoresis - hemoglobins. In: SMITH. I. Chromatographic and electrophoretic techniques. Vol. II: Zone electrophoresis. Londres: William Heinemann, 1968.

HUISMAN, T.H.J.; VLIET, G. van; SEBENS, T. Sheep haemoglobins: Some genetic and physiological aspects of two different adult haemoglobins in sheep. Nature. 182(4629):171-172, 1958.

JILEK, A.F.; BRADLEY, R.E. Hemoglobin types and resistance to Haemonchus contortus in sheep. Am. J. Vet. Res. 30(10):1773-1778, 1969.

KASSAI, T.; FÉSÜS, L.; HENDRIKX, W.M.L.; TAKÁTS, Cs.; FOK, É.; REDL, P.; TAKÁCS, E.; NILSSON, R.; van LEEUWEN, M.A.W.; JANSEN, J.; BERNADINA, W.E.; FRANKENA, K. Is there a relationship between haemoglobin genotype and the innate resistance to experimental Haemonchus contortus infection in Merino lambs? Vet. Paras. 37:61-77, 1990.

LE JAMBRE, L.F. Anthelmintics and preserving their effectiveness. In: LE JAMBRE, F.L.; KNOX, M.R. Sustainable Parasite Control in Small Ruminants. Proccedings of a Workshop, Bogor, Indonesia, 22-25 april, 1996, p.151 - 159.

PETZL, M.L.; PRIMO-PARMO, S.L. Eletroforese de hemoglobina, haptoglobina, anidrase carbônica II e esterase em gel de amido de milho comercial (maizena). Ciência e Cultura. 31(8):895-896, 1979.

PRESTON, J.M.; ALLONBY, E.W. The influence of haemoglobin phenotype on the susceptibility of sheep to Haemonchus contortus infection in Kenya. Res. Vet. Sci. 26:140-144, 1979.

SEAB. Departemento de Economia Rural. Acompanhamento da Situação Agropecuária do Paraná - Prognóstico da Pecuária, Fev. 1997.

SOTOMAIOR, C.S. Estudo de caracteres que possam auxiliar na identificação de ovinos resistentes e susceptíveis aos helmintos gastrintestinais. Tese de Mestrado - Curso de Pós-Graduação em Ciências Veterinárias. Setor de Ciências Agrárias, Universidade Federal do Paraná. Curitiba, 1997. 
SOUZA, F.P. Contribuição para o estudo da resistência de helmintos gastrintestinais de ovinos (Ovis aries) aos anti-helmínticos, no Estado do Paraná. Setor de Ciências Agrárias, Universidade Federal do Paraná, Curitiba, 1997.

THOMAZ-SOCCOL, V.T.; SOTOMAIOR, C.; SOUZA, F.P.; CASTRO, E.A.; PESSÔA SILVA, M.C.; MILCZEWSKI, V. Occurence of resistance to anthelmintics in sheep in Paraná State, Brazil. Veterinary Record. 139:421-422, 1996.

WALLER, P.J. The development of anthelmintic resistance in ruminant livestock. Acta Tropica. 56:233-243, 1994.
WANG, S.; FOOTE, W.C.; BUNCH, T.D. Evolutionary implications of haemoglobin polymorphism in domesticated and wild sheep. Small Ruminant Research. 4:315-322, 1991.

WINDON, R.G.; DINEEN, J.K.; KELLY, J.D. The segregation of lambs into "responders" and "nonresponders": response to vaccination with irradiated Trichostrongylus colubriformis larvae before weaning. Int. J. Paras. 10(1):65-73, 1980.

YAZWINSKI, T.A.; GOODE, L.; MONCOL, D.J.; MORGAN, G.W.; LINNERUD, A.C. Haemonchus contortus resistance in straightbred and crossbred Barbados Blackbelly sheep. J. An. Sci. 51(2):279-284, 1980. 